\title{
Solvothermal method as a green chemistry solution for micro- encapsulation of phase change materials for high temperature thermal energy storage
}

\author{
Albert Ioan Tudor ${ }^{1}$, Adrian Mihail Motoc ${ }^{1}$, Cristina Florentina Ciobota ${ }^{1}$, Dan. Nastase Ciobota ${ }^{1}$, Radu Robert \\ Piticescu $^{1, *}$, and Maria Dolores Romero-Sanchez ${ }^{1,2}$ \\ ${ }^{1}$ National Institute for Nonferrous and Rare Metals-IMNR, Blvd. Biruintei no.102, Pantelimon, Ilfov, Romania \\ ${ }^{2}$ Instituto Tecnologico del Calzado y Conexas-INESCOP, Elda-Alicante, Spain
}

Received: 15 November 2017 / Accepted: 18 January 2018

\begin{abstract}
Thermal energy storage systems using phase change materials (PCMs) as latent heat storage are one of the main challenges at European level in improving the performances and efficiency of concentrated solar power energy generation due to their high energy density. PCM with high working temperatures in the temperature range $300-500{ }^{\circ} \mathrm{C}$ are required for these purposes. However their use is still limited due to the problems raised by the corrosion of the majority of high temperature PCMs and lower thermal transfer properties. Micro-encapsulation was proposed as one method to overcome these problems. Different microencapsulation methods proposed in the literature are presented and discussed. An original process for the microencapsulation of potassium nitrate as PCM in inorganic zinc oxide shells based on a solvothermal method followed by spray drying to produce microcapsules with controlled phase composition and distribution is proposed and their transformation temperatures and enthalpies measured by differential scanning calorimetry are presented.
\end{abstract}

Keywords: phase change materials / thermal energy storage / micro-encapsulation

\section{Introduction}

Due to the advantages offered by latent heat thermal energy storage, such as low temperature variation during charging and discharging cycles, small unit size, high storage density, relatively constant heat transfer fluid (HTF) temperature during the discharge process and versatility, the use of PCMs as energy storage materials is growing in many different applications. In particular, PCMs with melting temperatures between 300 and $500{ }^{\circ} \mathrm{C}$ are needed in the storage of the heat obtained from high temperature concentrated solar thermal power plants. Micro-encapsulation of high temperature PCMs is a key process in reducing corrosion and improving thermal stability but problems related to thermal stability, mechanical resistance due to volume change during thermal cycling and compatibility between PCMs and encapsulation materials must be overcome.

\footnotetext{
* e-mail: rpiticescu@imnr.ro
}

\section{Actual state of thermal energy storage using high temperature PCMs}

Development of green energy solutions and achieving the $20 \%$ energy savings by 2020 using energy storage systems is one of the main objective of the European Energy Strategy 2020 [1]. Key energy storage technologies can be classified in two main categories depending on the energy production method: chemical energy storage and thermal energy storage [2]. Thermal energy systems (TES) developed using these technologies are classified in three groups: i) sensible heat storage that is based on storing thermal energy by heating or cooling a liquid or solid storage medium (e.g. water, sand, molten salts, rocks), with water being the cheapest option; ii) latent heat storage using phase change materials (PCMs) (e.g. from a solid state into a liquid state); and iii) thermo-chemical storage (TCS) using chemical reactions to store and release thermal energy. In Table 1 the advantages and disadvantages of these three types of thermal energy systems are presented. Developers from more European countries work on thermal energy storage (TES) systems for buildings and transportation of thermal energy [3]. 
Table 1. Advantages and disadvantages of TES [3].

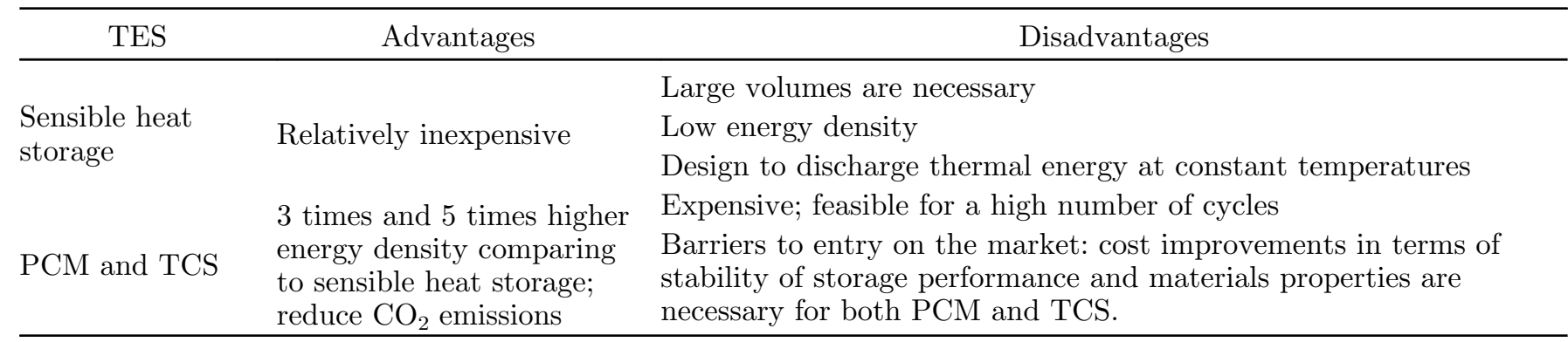

However, costs, performance and reliability should be verified, since these applications are at the beginning of commercialization. A thermal energy storage system combined with the existing equipment cooling capacity, for example, could be a solution for energy savings instead of adding more mechanical and electrical equipment like chillers, pumps, condensing equipment [4].

Different problems related to thermal stability and mechanical resistance due to volume change during thermal cycling, compatibility between PCMs and encapsulation materials and easiness of the encapsulation process, must be overcome.

Due to the advantages offered by latent heat thermal energy storage, such as low temperature variation during charging and discharging cycles, small unit size, high storage density and relatively constant HTF temperature during the discharge process, the use of PCMs as energy storage materials is growing in many different applications, thanks also to the versatility offered by the different melting temperature range.

In particular, $\mathrm{PCM}$ s with melting temperatures between 300 and $500{ }^{\circ} \mathrm{C}$ are required to be used for the storage of the heat obtained from high temperature concentrated solar thermal power plants.

Concentrated solar power (CSP) provides electricity relying on fossil or nuclear fuels (as most other technologies). They generate medium to high temperature heat, which is then used to operate a conventional power cycle through a steam or gas turbine.

CSP is a technology which produces electricity by concentrating solar energy in a single focal point, while producing no greenhouse gas emission, so it could be a key technology for mitigating climate change. This concentrated energy is then used to heat up a fluid, produce steam and activate turbines that produce electricity. The use of thermal energy storage technology solves the time mismatch between solar energy supply and electricity demand. When CSP combines with thermal storage capacity of several hours of full-capacity generation, CSP plants can continue to produce electricity even when clouds block the sun, or after sundown or in early morning when power demand steps up [5].

Thermal storage increases the value of the plant by providing guaranteed capacities. Storage can be used to extend the electricity generation after sunset, when electricity loads remain high [6]. Storage could also serve round-the-clock, base-load generation, displacing, e.g. high- $\mathrm{CO}_{2}$ emitting coal plants.
Simple and non-polluting CSP technologies can be deployed relatively quickly and can contribute substantially to reduce carbon dioxide emissions. Current CSP power plants are proving to be efficient and cost-effective. Today, sensible heat materials in the form of synthetic oil and molten salt are the most widely used storage materials in large-scale CSP systems with limitations commented above, while systems that utilize latent heat or thermochemical still being developed [7].

Innovation in energy technology is crucial to meet climate mitigation objectives while also supporting economic and energy security objectives. Cost-effective technologies are what will make the energy system transformation possible. However, continued dependence on fossil fuels and recent trends such as unexpected energy market fluctuations reinforce the role of governments, individually and collectively, to stimulate targeted action to ensure that resources are optimally aligned to accelerate progress.

Last researches and developments in the solar energy sector by different authors indicate the necessity to reduce the high costs of the operation and maintenance of the solar plants. In this sense, one of the most important research lines is addressed to the development and characterization of new PCMs to be used for energy storage. It is necessary to improve TES materials to build less expensive and more profitable solar plants, mainly avoiding corrosion phenomena at high temperatures, due to the high temperature conditions necessary to maintain the mixtures flowing, as well as the storage temperature, around $390^{\circ} \mathrm{C}$ in parabolic trough collectors and $565^{\circ} \mathrm{C}$ in central receivers $[8,9]$.

Although thermal energy storage is a key issue for CSP, the available and mature technologies of TES do not match all the actualized criteria for the properties required. As studied by Guillot et al. [10], alternative approaches have to be identified and developed to guaranty the expected extension of CSP implementations with respect to the International Energy Agency (IEA) 2050 scenario.

Energy Research Knowledge Centre (ERKC), funded by the European Commission to support its Information System of the Strategic Energy Technology Plan (SETIS) includes in its report CSP 2013, the 9 Priority Areas for the Energy sector. R\&D challenges in TES for CSP plants, according to ERKC include: increasing temperature limits, both the higher and lower limits, since most HTFs (e.g., molten salt mixtures) have a high melting point; maximizing heat transfer properties; enhancing compatibility with containing materials (e.g., tank walls); exploit- 
ing new material properties (e.g., phase change behaviour of mixtures, nanoparticles); increasing the life of materials subject to cyclic thermal loads (e.g., concretes); introducing new forms of storage (e.g., thermochemical reactions); reducing the overall costs of TES capital expenditure of commercial systems in the range of $25-40 \mathrm{EUR} / \mathrm{kWh}$. Table 2 includes a list of the last representative projects funded by the EU having as main objective the thermal energy storage in solar plants working in high temperatures range.

Additional alternative thermal energy storage approaches are needed, but they have to fulfil a list of essential properties such as: low cost, high availability, long life time expectancy, thermal stability up to $1000^{\circ} \mathrm{C}$, large storage capacity and good thermal conductivity, easy to be used, low $\mathrm{CO}_{2}$ emissions and renewable energy content. For latent heat storage, the high temperature levels of the future CSP plants $\left(200-1000^{\circ} \mathrm{C}\right)$ require the study of PCM (inorganic salts) with good stability at high temperature and high specifically heat. However, considering that the corrosion produced by molten salts could be significant, micro-encapsulation of high temperature PCMs is proposed as a solution to reduce/avoid corrosion problems.

In the next few years, and according to the IEA Solar PACES Implement Agreement, research and development efforts will be supported (mainly by the EU) for improvements in all components of CSP plants. The focus will be on troughs with direct steam generation, increasing the overall efficiency and development of Phase-change materials and concrete for thermal storage. Also for towers many innovative designs are currently proposed, with heat fluids and storage options. Currently, the use of PCMs as latent heat materials is still reduced and consequently its investment cost too.

For the next years, IEA points out an increase in the development of CSP based on the technologies offering new opportunities for energy storage. Typically, storage represents about one fifth of the total cost of a CSP plant with storage.

PCMs are products with a high potential for thermal management solutions in thermal energy storage systems (TES) due to their capacity to store and release thermal energy during the melting and crystallization process [11]. The main requirements for these materials can be grouped in three distinct categories: physical, technical and economical [12]. The temperature of the phase transformation and the higher melting enthalpy represent two of the most important requirements that a PCM must fulfil to store and to release heat. Depending on the working temperature range where PCMs must be used in TES, different organic, inorganic or metallic PCMs have been proposed. For high tempearute range, inorganic salts were reported as best candidates. The main storage properties of some PCMs are presented in Table 3 [13-20].

\section{Encapsulation of high temperature PCMs}

Encapsulation of PCM may increase their exchange surface required for a good heat transfer [21,22]. Moreover, it is important to notice that micro-encapsulated PCMs can store $10-15 \%$ more energy per unit volume than current molten salt systems. The possibility of using microencapsulated PCMs with different melting temperatures also increases efficiency of the energy transfer and a reduction of the residual waste heat.

Though macro-encapsulation $(>1 \mathrm{~mm})$ of high temperature PCMs has been reported by different authors in literature, nothing has been established to simultaneously solve the three problems encountered for the encapsulation of high temperature PCMs compared to low temperature PCMs: (1) liquid metal and molten salt generally exhibit high chemical corrosion in the presence of some shell materials (i.e. metals); (2) PCMs with solid to liquid transitions commonly show volume expansion and consequently they suffer thermal stress; (3) to reduce these problems, thick shells are used producing the drop of heat storage density [23].

An interesting study, carried out by the Lehigh University [24], about high temperature PCMs macroencapsulations, proposes $\mathrm{Zn}$ or $\mathrm{Al}$ as core materials macroencapsulated into stainless steel containers. Although software simulations with these systems demonstrate the thermal energy storage capacity of the PCMs, they also show a decrease in the latent heat storage capacity with thermal cycles or after long-term exposure to high temperatures. The reason seems to be the inter-metallic diffusion between the metal materials and the encapsulation metals during high temperature melting/solidification process. Thus, it is conceivable that metal PCMs materials macro-encapsulated by certain materials such as Al or steel are not suitable for TES. Using electroplating method, other authors have obtained particles ( $3 \mathrm{~mm}$ diameter) with lead-nickel core-shell structure suitable for heat recovery of high temperature waste heat [25].

More simple methods can also be used for the encapsulation of metal PCMs such as indium (melting temperature $=156{ }^{\circ} \mathrm{C}$ ) by using silica as shell by sol-gel procedure and obtaining nm size particles [26]. However, this method requires the melting of the metal, with increased difficultness for handling when using metals with higher melting temperature. $\mathrm{LiNaCO}_{3}$ has been prepared as eutectic by Ge et al. [27] to be used as PCM with melting temperature ca. $500^{\circ} \mathrm{C}$. Carbon allotropes have been used to enhance the thermal conductivity, finally obtaining composites with good physical and chemical stability and high thermal conductivity. Sol-gel processes using organic polymers with high melting temperature (i.e. polyimide) and organic-inorganic hybrids shells are proposed for the micro-encapsulation of PCMs in this project. For example, in this sense, stainless steel has been coated by sol-gel procedure of hybrid coatings for corrosion protection with interesting results, forming a physical barriers towards corrosion $[28,29]$.

Storing thermal energy in PCMs, such as inorganic salt mixtures, latent heat of fusion can increase the energy density for storage by $50 \%$. However, a major issue that has prevented the commercial use of PCM-TES for CSP is that it is difficult to discharge the latent heat stored in the PCM melt at specified heat rates. This is because when heat is extracted, the PCM-melt which has low thermal conductivity solidifies onto the heat exchanger surface decreasing 
Table 2. List of some research projects funded by the European Commission related with PCM thermal energy storage.

\begin{tabular}{l} 
Project \\
\hline Energy Storage for \\
Direct Steam Solar \\
Power Plants \\
(DISTOR)
\end{tabular}

Novel Efficient Solid

Storage for H2 (NESSHY)

FP6 Integrated

project. SES6-518271

(2006-2011)

New Solar Collector

Concept for High

Temperature Operation

in CSP applications

(HITECO)

Innovative configuration

for a fully renewable

hybrid CSP plant

(HYSOL)

FP7 2010-2013.

Grant agreement: 256830

FP7-ENERGY-20121-2STAGE. Grant agreement: 308912

(2013-2016)

High temperature thermal energy storage by

FP7-ENERGY-20111. Grant agreement: Reversible thermochemical 282677 (2012-2016) Reaction (STORRE)

Redox Materials-based Structured Reactors/Heat Exchangers for ThermoChemical Heat Storage Systems in Concentrated Solar Power Plants (RESTRUCTURE)

Concentrated Solar Power in Particles (CSP2)

Thermochemical Energy Storage for Concentrated Solar Power Plants (TCSPOWER)

Optimization of a thermal FP7-ENERGY-2011energy storage system with 1 . Grant agreement: integrated steam generator 283138 (2011-2014) (OPTS)

SAM.SSA - Sugar Alcohol FP7-ENERGY-2011based Materials for Seasonal Storage Applications
FP7-ENERGY-20111. Grant agreement: $283015(2011-2015)$

FP7-ENERGY-20111. Grant agreement: 282932 (2011-2015)

FP7-ENERGY-20111. Grant agreement: $282889(2011-2015)$
2. Grant agreement: $296006(2012-2015)$
Description

Development of thermal storage systems using phase change materials $(\mathrm{PCM})$ in the temperature range from $230{ }^{\circ} \mathrm{C}$ to $330^{\circ} \mathrm{C}$ for systems using steam between 30 and 100 bar

The project aims at developing novel materials, storage methods and fabrication processes that provide the energy density and the charge/discharge, storage/restitution rates necessary for mobile applications with spin-offs in stationary systems. To identify the most promising solid storage solutions for such applications.

The project is based on the research and development of a solar receiver that will increase the parabolic-trough efficiency, reaching an operating temperature of the heat transfer fluid above the current limit of $400^{\circ} \mathrm{C}$

Renewable energies have often problems in order to provide a stable and reliable power supply, as they often depend on meteorological circumstances that have a variable or stochastic component. This fact is often used by their detractors to favour the use of other alternatives such as fossil fuels.

Proposal concerns the area of thermal energy storage by chemical reaction for CSP. The objective is to develop a new solution for the heat storage with the following characteristics: Mid-term to long-term heat storage; High storage density; High temperatures $\left(300-550^{\circ} \mathrm{C}\right)$, which are representative of the CSP plants with cylinder-parabolic or CLFR technologies

Thermo chemical storage (TCS) involves the exploitation of the heat effects of reversible chemical reactions for the "storage" of solar heat. Among gas-solid reactions proposed for such an approach the utilization of a pair of redox reactions involving multivalent solid oxides has several inherent advantages that make it attractive for large-scale deployment

The CSP2 project puts forward an alternative heat transfer fluid (HTF) for concentrating solar power (CSP) plants. The use of dense gas-particle suspensions in tubes as HTF is proposed

The objective is to realise a new, efficient, reliable and economic thermochemical energy storage (TCS) for CSP, with the capability to contribute significantly to further cost reduction of regenerative electricity production. This will be achieved by applying reversible gas-solid reactions. Dissociation of calcium hydroxide is used for storing thermal energy in 450 and $550{ }^{\circ} \mathrm{C}$ OPTS project aims at developing a new TES system based on single tank configuration using stratifying Molten Salts as heat storage medium at $550{ }^{\circ} \mathrm{C}$, integrated with a Steam Generator (SG), to provide efficient, reliable and economic energy storage for the next generation of trough and tower plants

The project aims at developing new PCM for thermal energy seasonal storage applications in the range of medium temperatures. The generated materials provide: -Low cost, environmentally sound and safe solutions for seasonal storage applications, -Easy adjustment of the melting point for optimal "tuning" to the required applications, -Energy densities $>200 \mathrm{kWh} / \mathrm{m} 3$ for compact storage 
Table 3. Storage properties of inorganic high temperature PSMs.

\begin{tabular}{llll}
\hline Nr. Crt. & \multicolumn{1}{c}{ Compound } & Melting temperature $\left[{ }^{\circ} \mathrm{C}\right]$ & Latent heat $[\mathrm{KJ} / \mathrm{Kg}]$ \\
\hline 1 & $\mathrm{MgCl}_{2}{ }^{*} 6 \mathrm{H}_{2} \mathrm{O}$ & 117 & $165-168,6$ \\
2 & $\mathrm{NaOH}^{2}$ & 64,3 & 227,6 \\
3 & $\mathrm{LiNO}_{3}$ & 254 & 360 \\
4 & $\mathrm{KNO}_{3}$ & 333 & 266 \\
5 & $\mathrm{MgCl}_{2}$ & 714 & 452 \\
6 & $\mathrm{NaCl}$ & 800 & 492 \\
7 & $\mathrm{NaCO}_{3}$ & 854 & 276 \\
8 & $\mathrm{KF}$ & 857 & 452 \\
9 & $\mathrm{~K}_{2} \mathrm{CO}_{3}$ & 897 & 236 \\
10 & $\mathrm{KOH}_{12}$ & 380 & 145 \\
12 & $67 \% \mathrm{KNO}_{3}+33 \% \mathrm{LiNO}_{3}$ & 133 & 170 \\
13 & $54 \% \mathrm{KNO}_{3}+46 \% \mathrm{NaNO}_{3}$ & 222 & 100 \\
\hline
\end{tabular}

Table 4. Typical parameters of TES.

\begin{tabular}{llllll}
\hline \multicolumn{1}{c}{ TES } & Capacity $(\mathrm{kWh} / \mathrm{t})$ & Power $(\mathrm{MW})$ & Efficiency $(\%)$ & Storage period $(\mathrm{h}, \mathrm{d}, \mathrm{m})$ & Cost $(€ / \mathrm{kWh})$ \\
\hline Sensible & $10-50$ & $0.001-10$ & $50-90$ & $\mathrm{~d} / \mathrm{m}$ & $0.1-10$ \\
PCM & $50-150$ & $0.001-1$ & $75-90$ & $\mathrm{~h} / \mathrm{m}$ & $10-50$ \\
Chemical reactions & $120-250$ & $0.01-1$ & $75-100$ & $\mathrm{~h} / \mathrm{d}$ & $8-100$ \\
\hline
\end{tabular}

the heat transfer, and requiring large heat transfer area and hence a higher cost. Considering the technical problems encountered with the use of PCMs, micro-encapsulated PCMs may be an optimal solution for TES.

In order to establish the economic impact of the project in case of adaption of the developed micro-encapsulated PCMs to a CSP plant, a balance between the additional cost due to micro-encapsulation process and advantages produced in terms of energy efficiency and cost reduction in maintenance works, possible elimination of heat exchangers and/or HTFs, reduction on the required amount of PCMs, etc.

Macro $(>1 \mathrm{~mm})$ and micro-encapsulation properties (particle sizes of $\mu \mathrm{m}$ or $\mathrm{nm}$ ) have been successfully developed and patented for low temperature of PCMs (organic and inorganic materials) using mainly polymeric coatings [30-35]. In some literature papers and even pilot TES installations, macro-encapsulation of inorganic salts was used. $\mathrm{Zn}, \mathrm{NaNO}_{3}, \mathrm{MgCl}_{2}$ and eutectic mixtures having melting temperatures higher than $300{ }^{\circ} \mathrm{C}$ have been encapsulated according to patent US2011/0259544 [36] in which cylinders of materials base of $\mathrm{Ni}$ or carbon and stainless steel were used dimensions of $\mathrm{mm}$ to $\mathrm{cm}$. US patent no. $0055661 / 2012$ [37] refers to salt nitrates melted in metallic tubes which are sealed for permanent isolation. Recently, another method has been patented for encapsulating melted salts (US 2015/0284616) [38] such as $\mathrm{NaNO}_{3}$ or $\mathrm{KNO}_{3}$. This method is based on the coating of PCM pellets $(27 \mathrm{~nm})$ with a flexible polymer followed by a metallic coating by non-electric and electrolytic processes (Ni, Cu, Zn, ZnFe alloys, etc.).

For a maximum economic benefit, the use of inorganic shells $\left(\mathrm{SiO}_{2}, \mathrm{ZnO}\right.$, graphite, etc. $)$ and different oxides and ceramics as shell materials for the micro-encapsulation of PCMs, which are not expensive raw materials, may be developed based on sol-gel and hydrolysis, which are simple and cost-effective technologies, easy to be scaled-up and with machinery and equipment which are already developed for the food and pharmaceutical industries [39].

Regarding costs, the use of PCMs for thermal energy storage as latent heat materials is usually more expensive that sensible heat storage materials. However, the storage efficiency of latent heat materials is higher (75-90\% efficiency versus 50-90\%) for sensible materials.

The economic viability of a TES depends heavily on application and operation needs, including the number and frequency of the storage cycles; the thermal cycling stability of the PCM microcapsules (and therefore, reduction of work maintenance, replacement of energy storage materials, loss of storage capacity, etc.) are necessary improvements in the stability of the storage performance, which is associated with energy storage material properties. The importance of the $R \& D$ required in the development of new energy storage systems can be reflected in Table 4. 


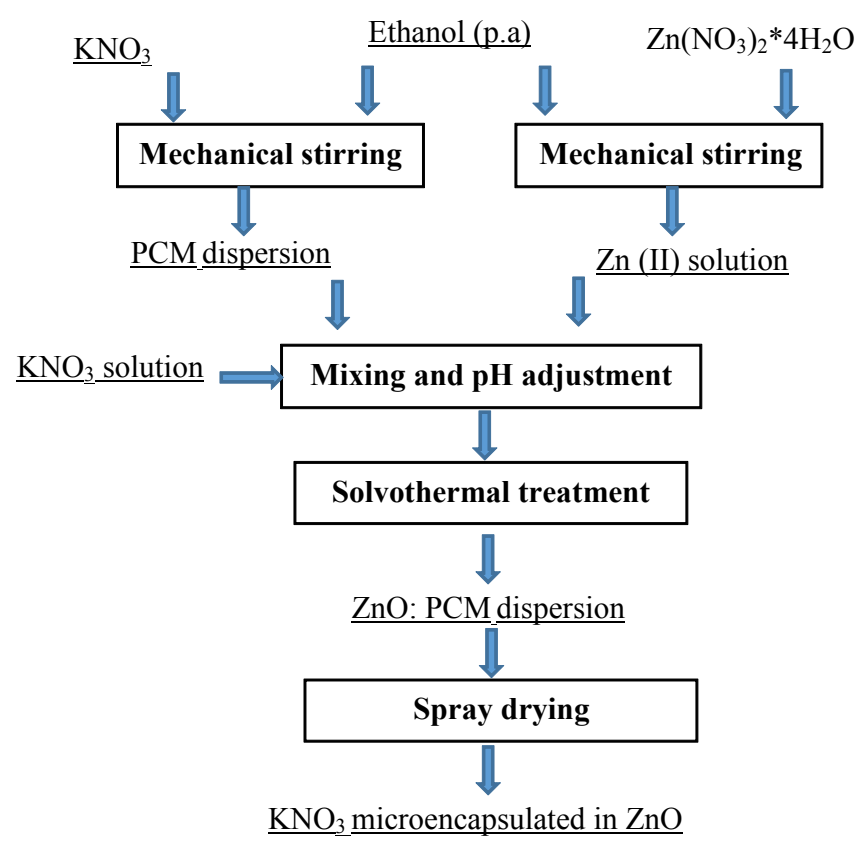

Fig. 1. Experimental procedure.

\section{Green solvothermal - spray drying method for PCMs micro-encapsulation in inorganic shell}

\subsection{Synthesis and characterization}

A green chemical process for micro-encapsulation of inorganic PCM salts such as $\mathrm{KNO}_{3}$ in $\mathrm{ZnO}$ shell was proposed with the aim to obtain encapsulated micro-spheres to be used as PCMs systems working in high temperature range, improve thermal stability and reduce TES corrosion produced by the molten salts. In the first step the shellforming material zinc nitrate tetra-hydrate $\left(\mathrm{Zn}\left(\mathrm{NO}_{3}\right)_{2} \times 4\right.$ $\mathrm{H}_{2} \mathrm{O}$ p.a. Merck) was dissolute in ethanol (p.a. grade) by mechanical stirring at room temperature in a glass reactor. A calculated weight of PCM salt was than dispersed under continuous stirring, according to a mass ratio $\mathrm{ZnO}$ : $\mathrm{PCM}$ in the range 1:1-1:5. A 3M KOH solution was then added under stirring until a $\mathrm{pH} \sim 9$ was reached. The suspension was than treated in a vertical autoclave endowed with TEFLON liner (CORTEST Inc., USA) for $2 \mathrm{~h}$ at 200 $250{ }^{\circ} \mathrm{C}$, under external Ar pressure of $40 \mathrm{~atm}$. The final suspension was granulated in a spray dryer at a constant feeding rate of $5 \mathrm{l}$ suspension/hour with different nozzle sizes (LabPlant, U.K.) and hot air at $100^{\circ} \mathrm{C}$ as drying agent. The optimal spray drying conditions were selected based on the measurement of their flowability using a Hall Flowmeter funnel according to ASTM B213-17. The as-obtained spray dried micro-granulated powder was sieved and powders with sizes in the range $20-50 \mu \mathrm{m}$ were characterized for determination of the chemical composition, phase composition and distribution and thermal behavior. The $\mathrm{K}$ and $\mathrm{Zn}$ contents were analyzed by atomic absorption spectroscopy (AAS ZEEnit 700, Karl Zeiss) and chemical titration according to the Romanian Standards 3223/1-92 and 1269/

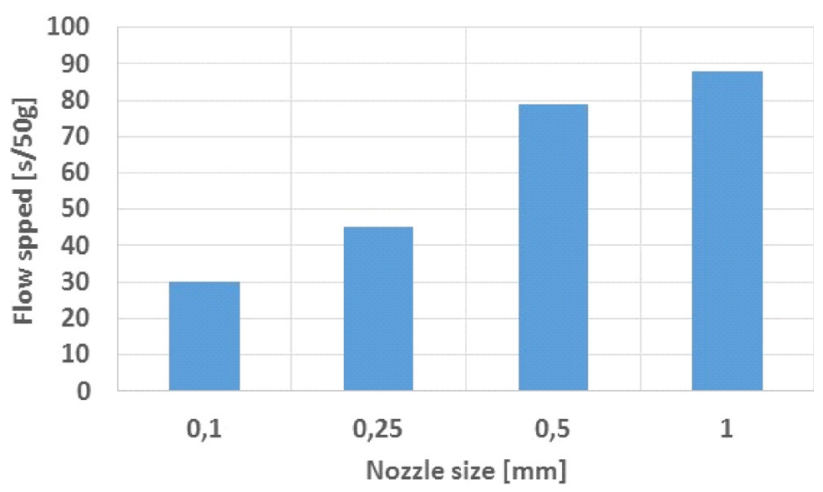

Fig. 2. Flowability of micro-encapsulated powders vs. spray drier nozzle size.

4-89 respectively. The experimental procedure is presented in the schematic flowsheet from Figure 1.

The phase composition was analyzed by XRD (Brucker D8 Advance) with the help of DIFFRAC ${ }^{+}$software by the Brag-Brentano method in $\theta-\theta$ coupling mode, using the $\mathrm{CuK} \alpha$ source in the range $2 \theta=4 \ldots 74^{\circ}$.

The thermal properties of the initial $\mathrm{KNO}_{3}$ salt and $\mathrm{KNO}_{3}$ micro-encapsulated in $\mathrm{ZnO}$ shell were measured by differential scanning calorimetry (DSC Netzsch 200 Maya F3) in the temperature range $-40 \ldots 600^{\circ} \mathrm{C}$. Samples of $10-$ $15 \mathrm{mg}$ of powders have been weight using a four decimal precision analytical balance. The samples were charged in special Al crucibles preview with $\mathrm{Al}$ perforated lead to avoid material losses. DSC spectra were recorded in Ar flow gas at a constant heating rate of $10 \mathrm{Kmin}^{-1}$.

Phase distribution and the formation of shell structure was analyzed by SEM -EDX method using a TESCAN -Vega II LMU unit.

\subsection{Results and discussion}

Spray drying is a key step in encapsulating the $\mathrm{KNO}_{3}$ salt as $\mathrm{PCM}$ phase in an inorganic $\mathrm{ZnO}$ shell with desired properties for thermal energy storage. The flowability of KNO3 microencapsulated in $\mathrm{ZnO}$ shell was selected to rapidly control the encapsulation process based on the assumption that powders with round shapes and homogeneous sizes have better flowability properties. During spray-drying process the best method that could be used to adjust the sizes and flowablility of microcapsules was to use nozzles with different sizes. It was observed that flowability of the microcapsules increases with increasing nozzle size from $0.25 \mathrm{~mm}$ to $1 \mathrm{~mm}$ The microcapsules obtained by spry drying using a $1 \mathrm{~mm}$ nozzle size have the highest flow speed (Fig. 2) and all microcapsules were further prepared in these conditions.

The solvothermal treatment temperature is the main parameter that influences the phase composition of the micro-encapsulated powder. According to the thermodynamic prediction done using the E-pH module of HSC 8.0 software for Zn-K-H-O system, it is expected that increasing the solvothermal temperature from room temperature (Fig. 3) to $250^{\circ} \mathrm{C}$ (Fig. 4), the formation of the metastable phase zinc hydroxi-nitrate with different $\mathrm{NO}_{3}: \mathrm{H}_{2} \mathrm{O}$ ratios is suppressed. 


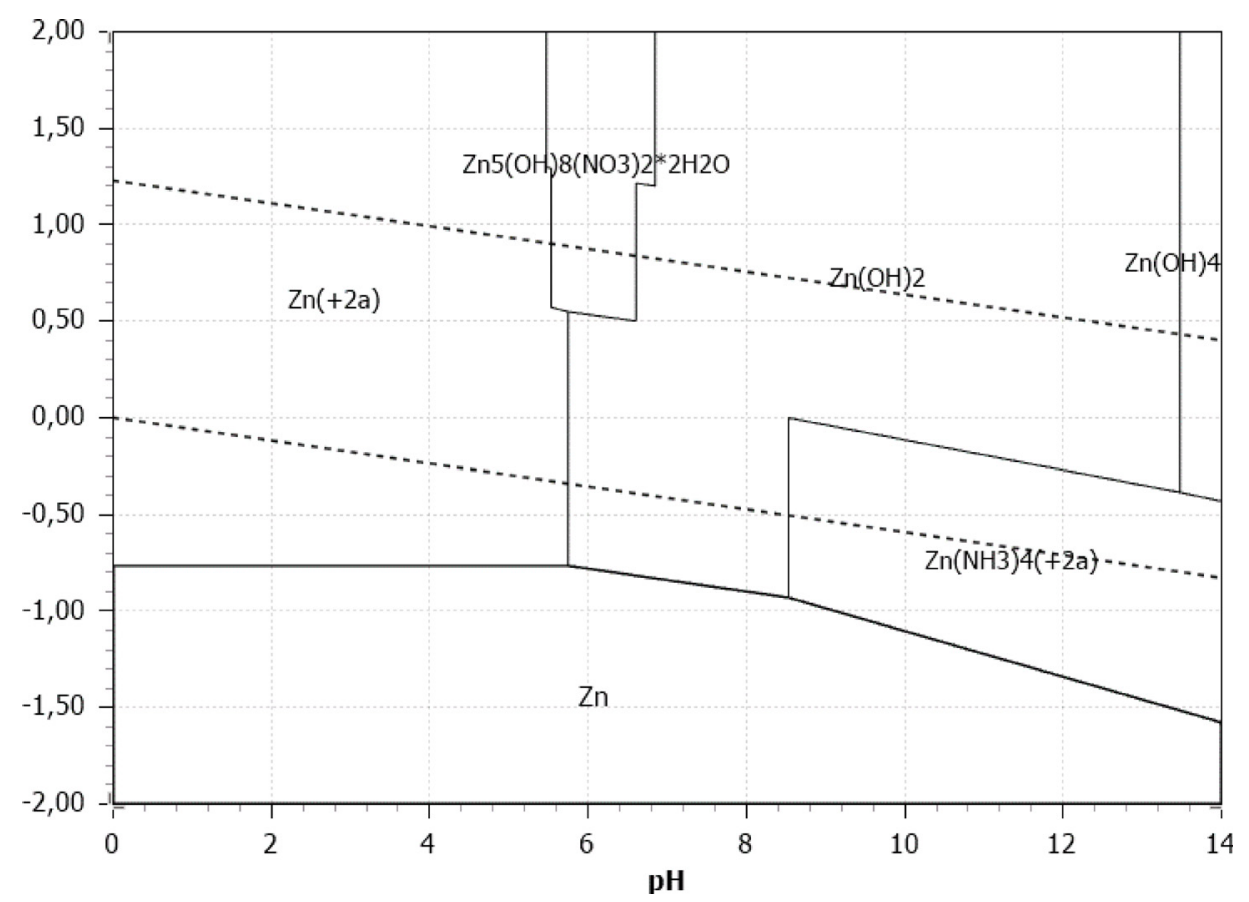

Fig. 3. E-pH (Pourbaix) diagram of Zn-K-O-H system at room temperature.

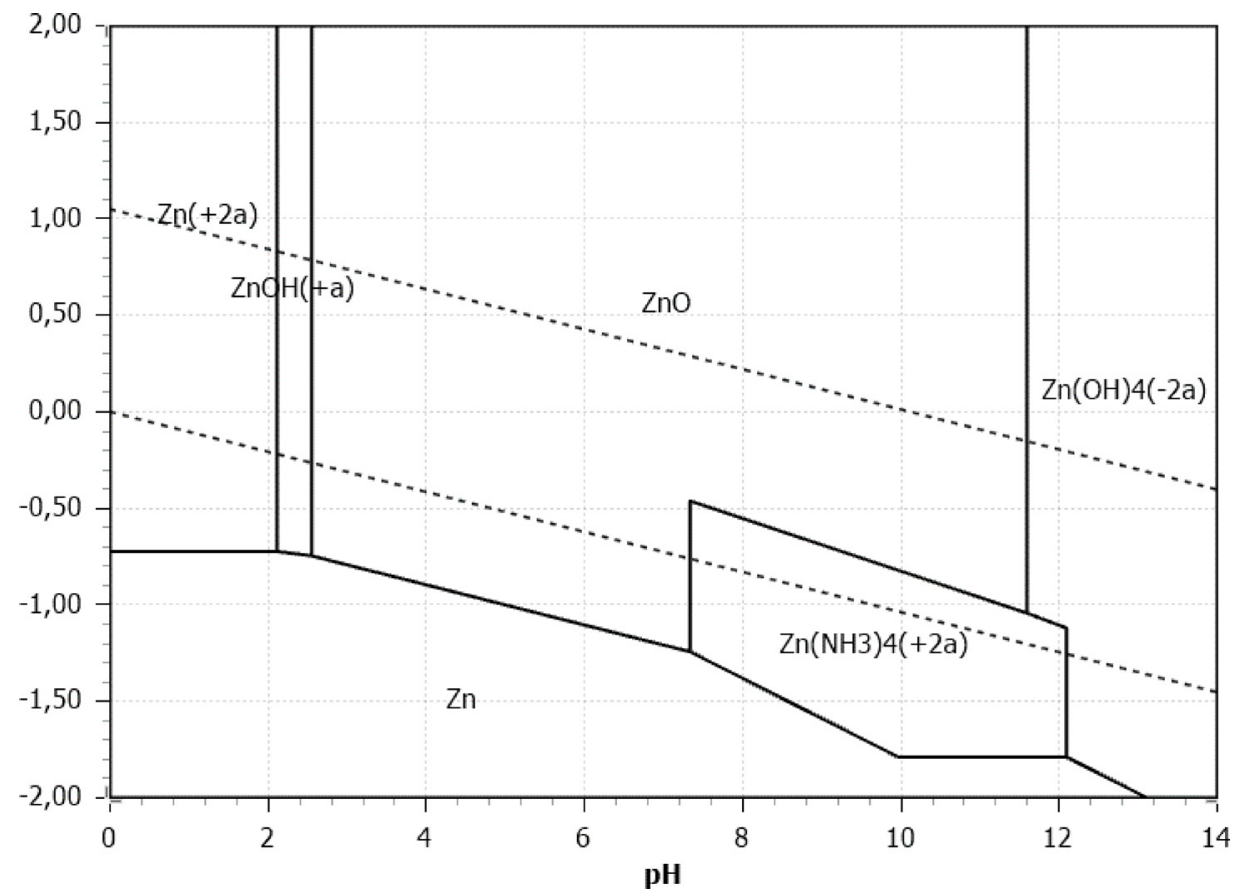

Fig. 4. E-pH (Pourbaix) diagram of $\mathrm{Zn}-\mathrm{K}-\mathrm{O}-\mathrm{H}$ system at $250^{\circ} \mathrm{C}$.

The XRD patterns of two selected spray-dried powders from $\mathrm{KNO}_{3}-\mathrm{ZnO}$ system obtained at the lowest experimental temperature $\left(110^{\circ} \mathrm{C}\right)$ and highest experimental temperature $\left(250^{\circ} \mathrm{C}\right)$ confirm these prediction, as seen in Figure 5. The results are summarized in Table 5 .
Figure 6 presents the SEM image of the spray dried sample P2. It may be observed the morphology of the powder consisting of core $\mathrm{KNO}_{3}$ irregular shape crystals covered by transparent flattened $\mathrm{ZnO}$ crystals. The EDX analysis from Figures 7 and 8 performed on the core and 


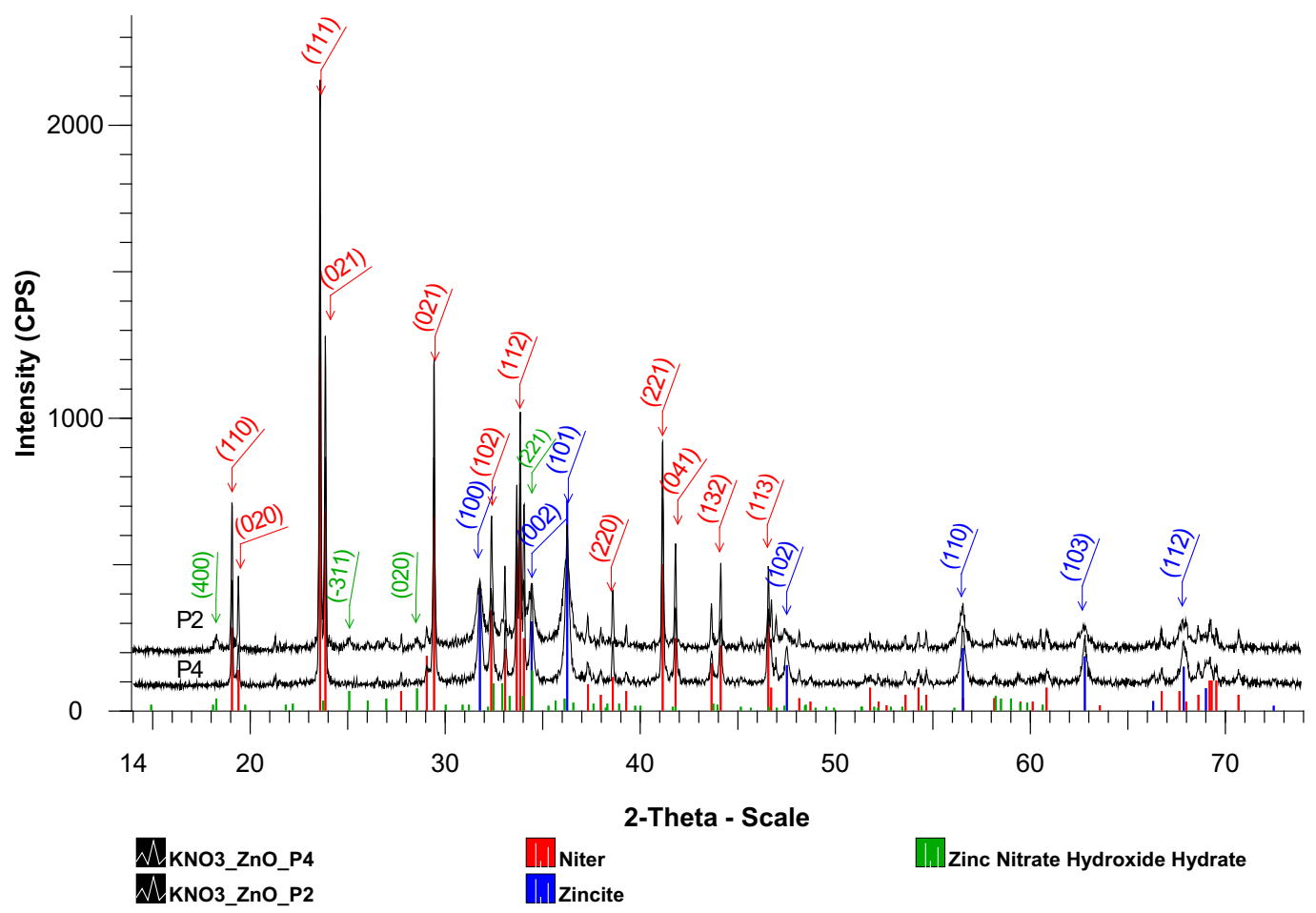

Fig. 5. XRD pattern of samples P2 and P4.

Table 5. Phase composition of $\mathrm{KNO}_{3}-\mathrm{ZnO}$ microcapsules with different weight ratios obtained at different solvothermal synthesis temperatures.

\begin{tabular}{|c|c|c|c|c|}
\hline $\begin{array}{l}\text { Sample } \\
\text { code }\end{array}$ & $\begin{array}{c}\mathrm{KNO}_{3}: \\
\mathrm{ZnO} \\
\text { ratio }\end{array}$ & $\begin{array}{c}\text { Synthesis } \\
\text { temperature, }{ }^{\circ} \mathrm{C}\end{array}$ & $\begin{array}{l}\text { Phases } \\
\text { detected }\end{array}$ & Wt.\% \\
\hline \multirow{3}{*}{$\mathrm{P} 1$} & \multirow{3}{*}{$1: 2$} & \multirow{3}{*}{110} & $\mathrm{KNO}_{3}$ & 76.1 \\
\hline & & & $\mathrm{ZnO}$ & 20.2 \\
\hline & & & $\begin{array}{l}\mathrm{Zn}_{5}(\mathrm{OH})_{8} \\
(\mathrm{NO} 3)_{2}(\mathrm{H} 2 \mathrm{O})_{2}\end{array}$ & 3.6 \\
\hline \multirow{3}{*}{ P1 TT } & \multirow{3}{*}{$1: 3$} & \multirow{3}{*}{150} & $\mathrm{KNO}_{3}$ & 51.6 \\
\hline & & & $\mathrm{ZnO}$ & 14.4 \\
\hline & & & $\begin{array}{l}\mathrm{Zn}_{5}(\mathrm{OH})_{8} \\
(\mathrm{NO} 3)_{2}(\mathrm{H} 2 \mathrm{O})_{2}\end{array}$ & 33.9 \\
\hline \multirow{3}{*}{ P2 } & \multirow{3}{*}{$1: 5$} & \multirow{3}{*}{110} & $\mathrm{KNO}_{3}$ & 63 \\
\hline & & & $\mathrm{ZnO}$ & 18 \\
\hline & & & $\begin{array}{l}\mathrm{Zn}_{5}(\mathrm{OH})_{8} \\
(\mathrm{NO} 3)_{2}(\mathrm{H} 2 \mathrm{O})_{2}\end{array}$ & 18 \\
\hline \multirow{2}{*}{ P3 } & \multirow[b]{2}{*}{$1: 5$} & \multirow{2}{*}{200} & $\mathrm{KNO}_{3}$ & 88.8 \\
\hline & & & $\mathrm{ZnO}$ & 11.2 \\
\hline \multirow{2}{*}{$\mathrm{P} 4$} & \multirow[b]{2}{*}{$1: 5$} & \multirow{2}{*}{250} & $\mathrm{KNO}_{3}$ & 79.5 \\
\hline & & & $\mathrm{ZnO}$ & 20.5 \\
\hline
\end{tabular}

shell components respectively shows clearly the bi-phasic composition with no inter-diffusion between the two components.

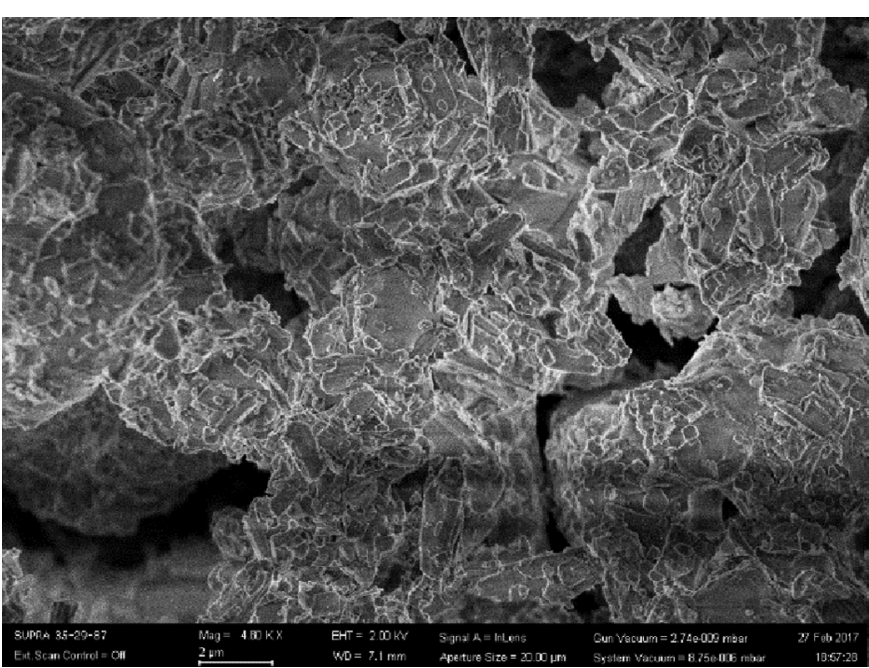

Fig. 6. SEM image of $\mathrm{KNO}_{3}-\mathrm{ZnO}-\mathrm{P} 2$ sample.

Thermal properties of the micro-encapsulated PCMs are the main properties enabling the future application of these materials in thermal energy storage. It is important to compare the phase transformation temperatures and related transformation enthalpies of pure and microencapsulated PCM for future utilization in thermal energy storage systems.

Figures 9-11 present the DSC spectra of initial pure $\mathrm{KNO}_{3}, \mathrm{KNO}_{3}-\mathrm{ZnO}-\mathrm{P} 2$ and $\mathrm{KNO}_{3}-\mathrm{ZnO}-\mathrm{P} 4$ micro-encapsulated samples. The calculated values of the melting and crystallization temperatures and enthalpies are summarized in Table 6 . 


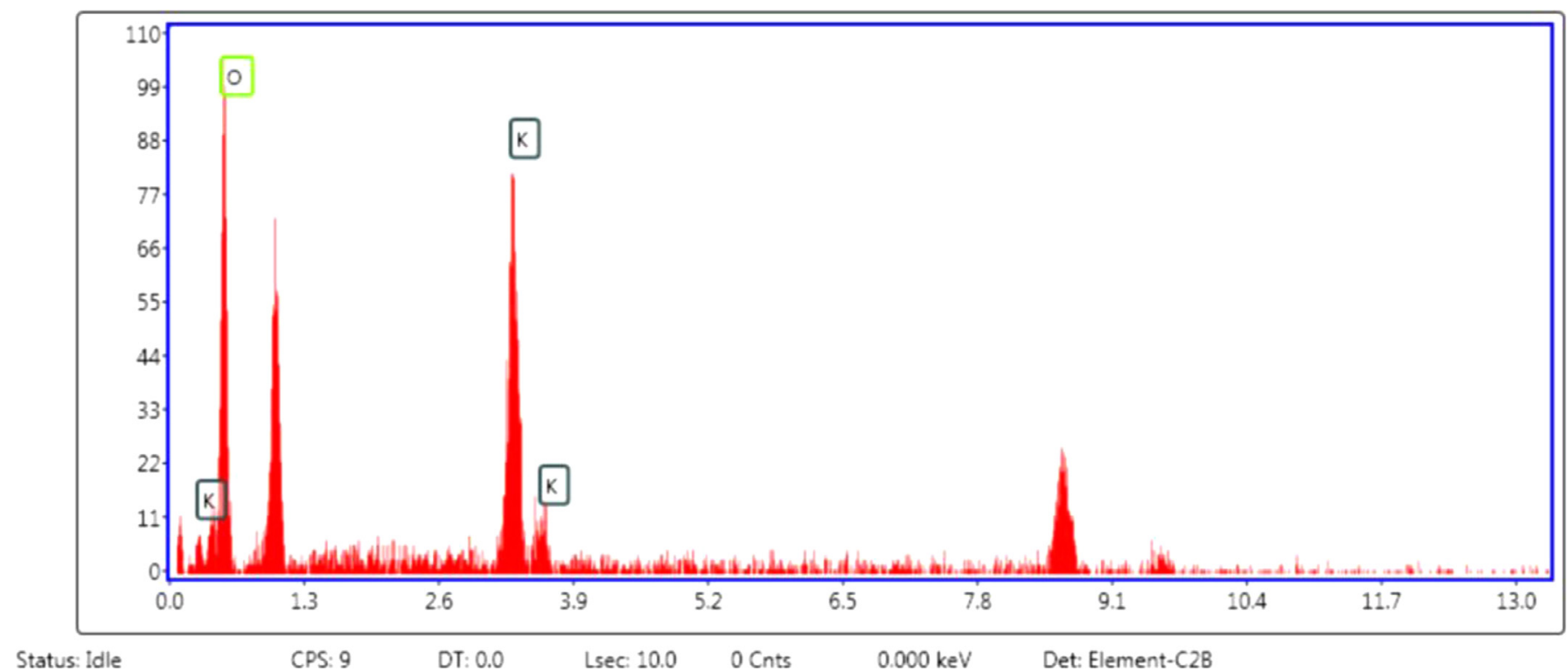

Fig. 7. EDX of a core region. The chemical composition corresponds to $\mathrm{K}=30.28$ wt.\% (15.09 at.\%) and O=69.72 wt.\% (84.91 at.\%).

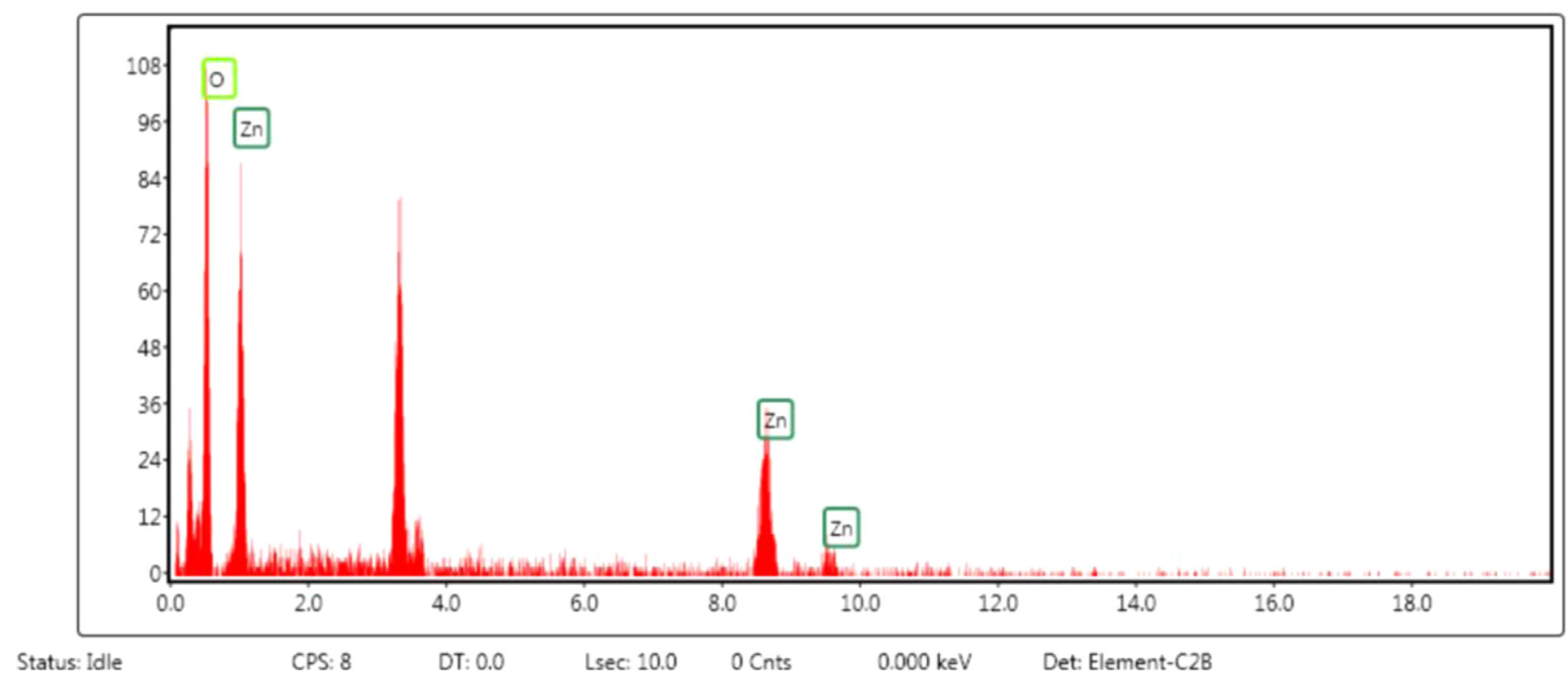

Fig. 8. EDX of a shell region. The chemical composition corresponds to $\mathrm{Zn}=39.03$ wt.\% (13.55 at. $\%)$ and $\mathrm{O}=60.97$ wt. $\%(86.45$ at. $\%)$.

From the experimental results presented it may be observed that micro-encapsulation of $\mathrm{KNO}_{3}$ in inorganic $\mathrm{ZnO}$ shell does not affect the PCM behavior of the $\mathrm{KNO}_{3}$ core material. The melting and crystallization temperatures are very similar for all samples. A decrease in the transformation enthalpies could be observed in the microencapsulated materials. The transformation enthalpies of sample $\mathrm{P} 4$ are about 2 times higher than values for sample $\mathrm{P} 2$; this may be explained by the presence of secondary zinc nitrate hydroxide hydrate $\left(\mathrm{Zn}_{5}(\mathrm{OH})_{8}(\mathrm{NO} 3)_{2}(\mathrm{H} 2 \mathrm{O})_{2}\right)$ phase. It may be concluded that solvothermal synthesis of $\mathrm{ZnO}$ shell at temperatures $>200^{\circ} \mathrm{C}$ ensures the optimal micro-encapsulation process for high temperature PCM based on $\mathrm{KNO}_{3}$ salt. Further experiments are in course to study the durability of the microcapsules by performing cyclic DSC measurements in conditions similar to real TES.

\section{Conclusions}

- Development of green energy solutions and achieving the $20 \%$ energy savings by 2020 using energy storage systems is one of the main objectives of the European Energy Strategy 2020. Thermal energy systems developed using latent heat storage using PCMs have as main advantage higher energy densities compared to traditional sensible heat storage materials but are more expensive and their 


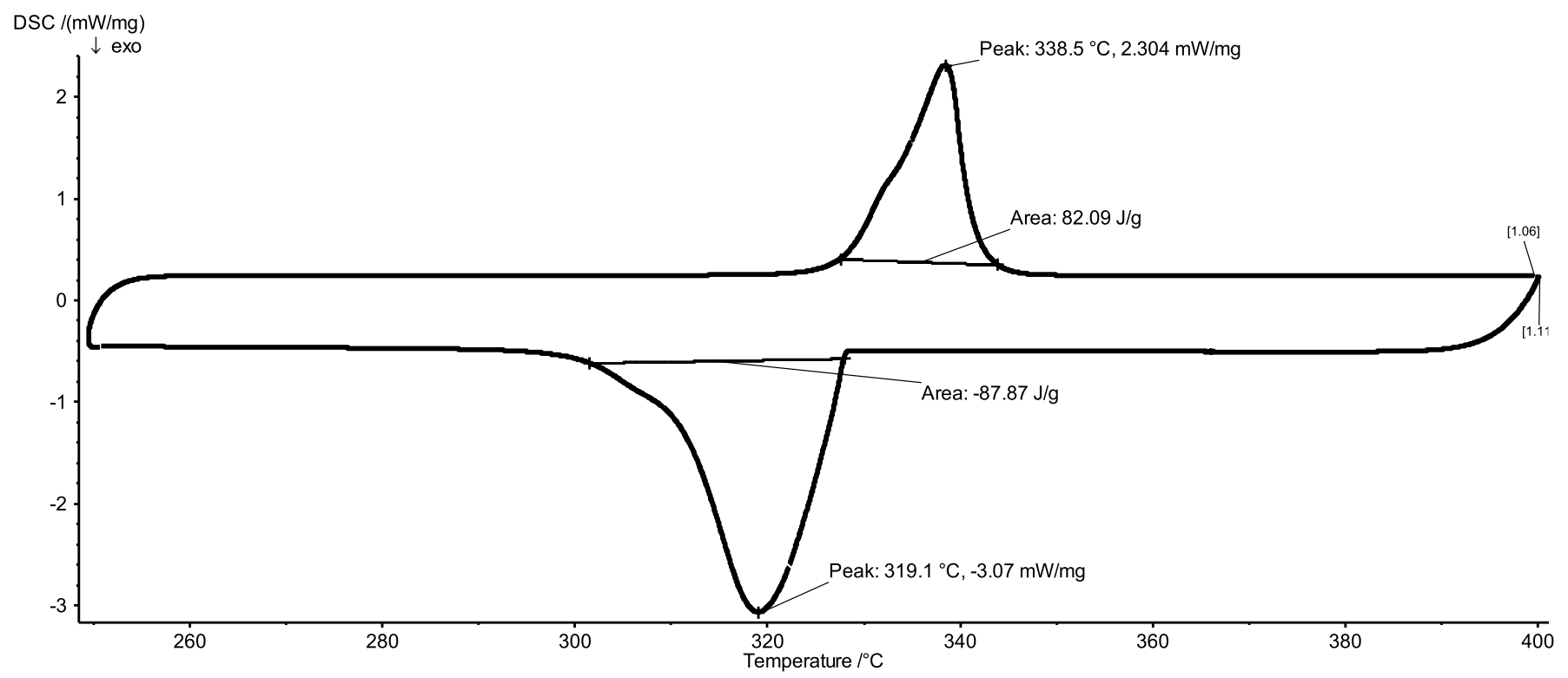

Fig. 9. DSC spectra of initial pure $\mathrm{KNO}_{3}$.

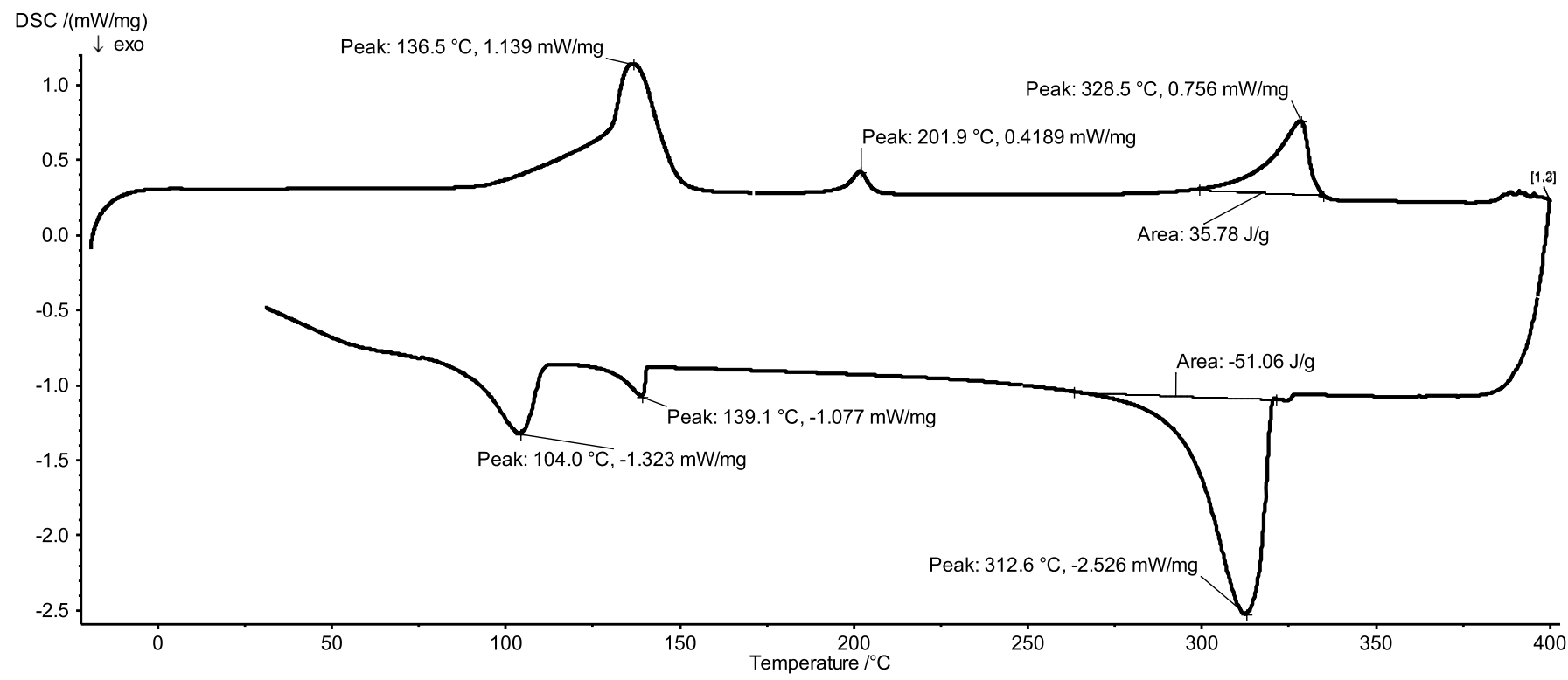

Fig. 10. DSC spectra of $\mathrm{KNO}_{3}-\mathrm{ZnO}-\mathrm{P} 2$ sample.

stability for a high number of cycles remain a barrier to entry on the market. For PCMs working at higher temperatures required for CSP stations, based mainly on inorganic salts such as nitrates, corrosion and thermal stability became crucial for application. One method to solve these problems is to encapsulate the inorganic PCM phase in a shell materials to avoid contact with the storage reactor, leading to reduced corrosion and better thermal stability;

- a combined solvothermal synthesis followed by spray drying process to micro-encapsulate $\mathrm{KNO}_{3}$-based PCM in an inorganic ZnO shell was for the first time proposed. Solvothermal synthesis in ethanol at temperatures up to $250^{\circ} \mathrm{C}$ lead to the formation of a bi-phasic system consisting only from the PCM and shell without any interactions between them. Microcapsules with different $\mathrm{ZnO}: \mathrm{KNO} 3$ ratios were developed by spray drying with hot air at $100{ }^{\circ} \mathrm{C}$ using a $1 \mathrm{~mm}$ size nozzle;

- the thermal characteristics of these core/shell microcapsules were analyzed by DSC showing the best results corresponds to micro-capsules obtained in solvothermal conditions avoiding the presence of secondary hydroxyzincite phases, without affecting the transformation temperatures and enthalpies of the micro-encapsulated PCM. Works are in progress to study the thermal stability for high number of cycles required for application. 


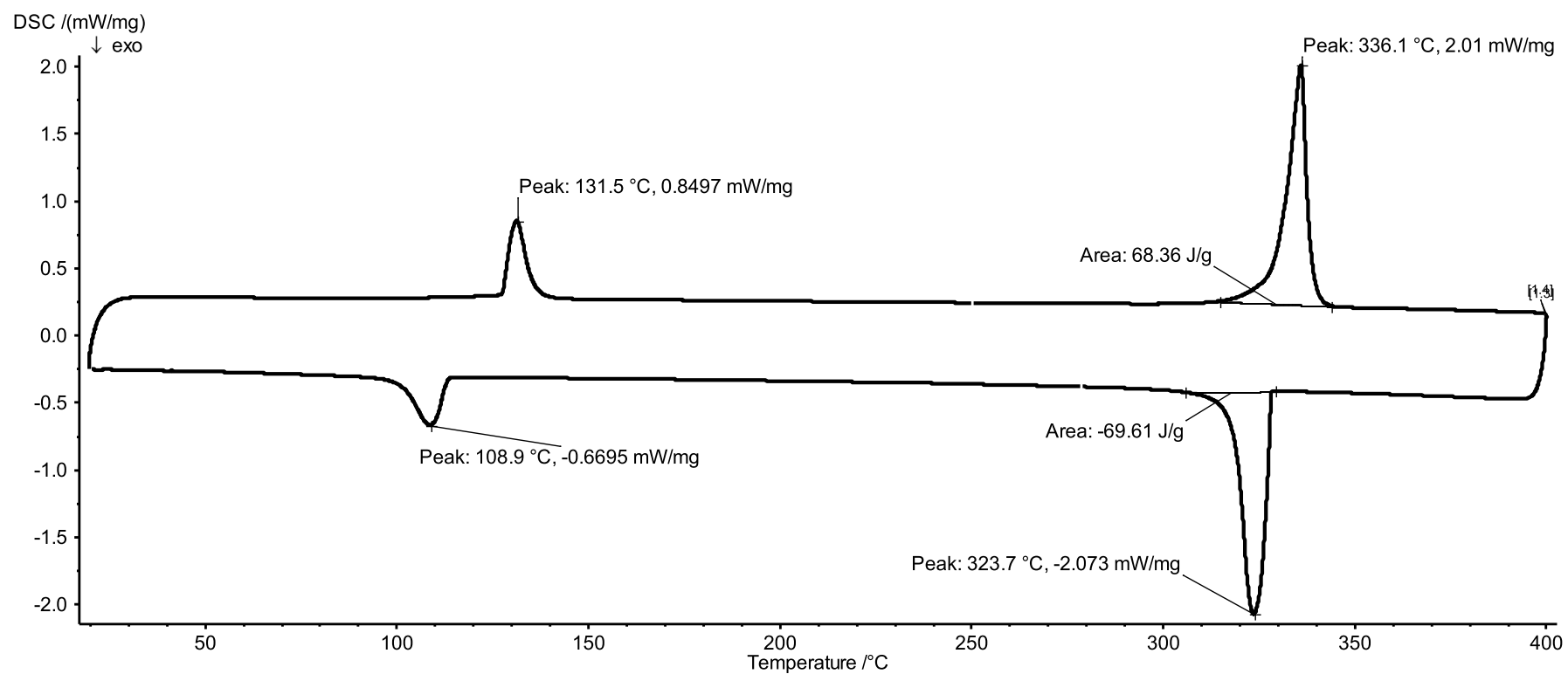

Fig. 11. DSC spectra of $\mathrm{KNO}_{3}-\mathrm{ZnO}-\mathrm{P} 4$ sample.

Table 6. Melting and crystallization temperatures and enthalpies of pure $\mathrm{KNO}_{3}$ and $\mathrm{KNO}_{3}$ micro-encapsulated in $\mathrm{ZnO}$ shell.

\begin{tabular}{llllc}
\hline \multicolumn{1}{c}{ Sample } & \multicolumn{3}{c}{ Melting } & \multicolumn{2}{c}{ Crystallization } \\
& $\mathrm{T}_{\mathrm{m}}\left({ }^{\circ} \mathrm{C}\right)$ & $\Delta \mathrm{H}_{\mathrm{m}}(\mathrm{J} / \mathrm{g})$ & $\mathrm{T}_{\mathrm{cr}}\left({ }^{\circ} \mathrm{C}\right)$ & 87.90 \\
\hline KNO3 & 338.5 & 82.09 & 319.1 & 51.06 \\
KNO3-ZnO P2 & 328.5 & 35.78 & 312.6 & 69.61 \\
KNO3-ZnO P4 & 336.1 & 63.36 & 323.7 & $\mathrm{~J} / \mathrm{g})$ \\
\hline
\end{tabular}

We gratefully acknowledge the financial support received from European Commission and Romanian Government-Management Authority from Ministry of Research and Innovation, in the frame of Competiveness Operational Programme, Action A1.1.4-E2015 , project ID P 37 776, SMIS code 104730, Acronym ENERHIGH.

\section{References}

1. A strategy for competitive, sustainable and secure energy, DG - Energy, 2011

2. I. Stefanescu, M. Varlam, E. Carcadea, New approaches on the energy storage technologies in Romania, Bul. AGIR/ Supl. 1 (2015) 54-60

3. Thermal Energy Storage/Technology Brief, IEA-ETSAP and IRENA@ Technology Brief E17-January 2013

4. P.E. Guy Frankenfiled, USA presentation

5. http://ec.europa.eu/research/energy/eu/index_en.cfm? $\mathrm{pg}=$ research-csp-support

6. Geyer, SolarPACES Annual Report 2007

7. S. Kuravi, J. Trahan, D. Yogi Goswami, M.M. Rahman, E.K. Stefanakos, Thermal energy storage technologies and systems for concentrating solar power plants, Prog. Energy Combust. Sci. 39 (2013) 285-319
8. A.G. Fernández, H. Galleguillos, F.J. Pérez, Corrosion ability of a novel heat transfer fluid for energy storage in CSP plants, Oxid. Met. 82 (2014) 331-345

9. J. Burgaleta, SENER torresol energy win U.S. CSP today awards, Renew. Energy Focus 12 (2010)

10. S. Guillot, A. Faik, A. Rakhmatullin, J. Lambert, E. Veron, P. Echegut, C. Bessada, N. Calvet, X. Py, Corrosion effects between molten salts and thermal storage materialfor concentrated solar power plants, Appl. Energy 94 (2012) 174-181

11. I. Dinçer, M.A. Rosen, Thermal energy storage systems and applications, John Wiley \& Sons, 2002

12. H. Mehling, L.F. Cabeza, Heat and cold storage with PCM. An up to date introduction into basics and applications, Springer, Berlin, Germany, 2008

13. V.V. Tyagi, D. Buddhi, PCM thermal storage in buildings: a state of art, Renew. Sustain. Energy Rev. 11 (2007) 1146-1166

14. H. Mehling, L.F. Cabeza, Phase change materials and their basic properties, in: Thermal Energy Storage For Sustainable Energy Consumption: Fundamentals, Case Studies And Design (Ed. H.O. Paksoy), Kluwer Academic Publishers Group, 2007, pp. 257-278

15. B. Zalba, J.M. Marín, L.F. Cabeza, H. Mehling, Review on thermal energy storage with phase change: materials, heat transfer analysis and applications, Appl. Therm. Eng. 23 (2003) 251-283 
16. A. Sharma, V.V. Tyagi, C.R. Chen, D. Buddhi, Review on thermal energy storage with phase change materials and applications, Renew. Sustain. Energy Rev. 13 (2009) 318345

17. T. Nomura, C. Zhu, N. Sheng, G. Saito, T. Akiyama, Microencapsulation of metal-based phase change material for hightemperature thermal energy storage, Sci. Rep. 5 (2015) 9117. DOI: $10.1038 /$ srep09117

18. W. Zhao, Characterization of encapsulated phase change materials for thermal energy storage, Theses and dissertations, Paper 1135 Lehigh University, 2013

19. A. Gil, M. Medrano, I. Martorell, A. Lazaro, P. Dolado, B. Zalba, L.F. Cabeza, State of the art on high temperature thermal energy storage for power generation. Part 1Concepts, materials and modellization, Renew. Sustain. Energy Rev. 14 (2010) 31-55

20. M. Medrano, A. Gil, I. Martorell, X. Potau, L.F. Cabeza, State of the art on high temperature thermal energy storage for power generation. Part 2-Case studies, Renew. Sustain. Energy Rev. 14 (2010) 56-72

21. A. Stein et al., Adv. Mater. 21 (2009) 265-293

22. C. Liang, et al., Angew. Chem. Int. Ed. 47 (2008) 3696-3717

23. T. Nomura, C. Zhu, N. Sheng, G. Saito, T. Akiyama, Microencapsulation of metal-based phase change material for hightemperature thermal energy storage, Sci. Rep. 5 (2015) 9117. DOI:10.1038/srep09117

24. W. Zhao, Characterization of encapsulated phase change materials for thermal energy storage, Theses and dissertations, Paper 1135 Lehigh University, 2013

25. N. Maruoka, T. Akiyama, Thermal stress analysis of PCM encapsulation for heat recovery of high temperature waste heat, J. Chem. Eng. Japan 36 (2003) 794-798

26. Y. Hong, S. Ding, W. Wu, J. Hu, A.A. Voevodin, L. Gschwender, E. Snyder, L. Chow, M. Su, Enhancing heat capacity of colloidal suspension using nanoscale encapsulated phase-change materials for heat transfer, Appl. Mater. Interfaces 2 (2010) 1685-1691

27. Z. Ge, F. Ye, H. Cao, G. Leng, Y. Qin, Y. Ding, Carbonatesalt-based composite materials for medium and hightemperature thermal energy storage, Particuology $\mathbf{1 5}$ (2014) $77-81$

28. P. Chou, C. Chandrasekaran, G.Z. Cao, Sol-gel derived hybrid coatings for corrosion protection, J. Sol-Gel Sci. Technol. 26 (2003) 321-327

29. R.B. Figueira, C.J.R. Silva, E.V. Pereira, Organic-inorganic hybrid sol-gel coatings for metal corrosion protection: a review of recent progress, J. Coat. Technol. Res. 12, 2015, 1-35

30. M. Graham, E. Shchukina, P. Felix De Castro, D. Shchukin, Nanocapsules containing salt hydrate phase change materials for thermal energy storage, J. Mater. Chem. A 4 2016, 16906 16912

31. D. Platte, U. Helbig, R. Houbertz, G. Sextl, Macromol. Mater. Eng. 298 (2013) 67-77

32. A. Schoth, K. Landfester, R. Muñoz-Espi, Langmuir 31 (2015) 3784-3788

33. F. Salaun, E. Devaux, S. Bourbigot, P. Rumeau, Carbohydr. Polym. 79 (2010) 964-974

34. Procedure for micro-encapsulation of phase change materials by spray-drying. EP 2119498 A1

35. US $2017 / 0030077$. Micro-encapsulation of materials using cenospheres

36. US $2011 / 0259544$. Encapsulated phase change apparatus for thermal energy storage

37. US 2012 /0055661. High temperature thermal energy storage system

38. US 2015 /0284616. Encapsulation of thermal energy storage media

39. A.M. Motoc, A.I. Tudor, M. Petriceanu, V. Badilita, E.J.P. del Barrio, Prasanta, V. Fierro, A. Celzard, R.R. Piticescu, Mater. Chem. Phys. 161 (2015) 219-227

Cite this article as: Albert Ioan Tudor, Adrian Mihail Motoc, Cristina Florentina Ciobota, Dan. Nastase Ciobota, Radu Robert Piticescu, Maria Dolores Romero-Sanchez, Solvothermal method as a green chemistry solution for micro-encapsulation of phase change materials for high temperature thermal energy storage, Manufacturing Rev. 5, 4 (2018) 